\title{
Impact of Tumor-Derived CCL2 on Macrophage Effector Function
}

\author{
M. S. Brault and R. A. Kurt* \\ Department of Biology, Lafayette College, Easton, PA 18042, USA
}

Received 16 January 2004; accepted 11 June 2004

\begin{abstract}
Monocyte chemoattractant protein-1 (MCP-1, CCL2) is produced by many different types of cells. In the current investigation, the effect of tumor-derived CCL2 on macrophages was evaluated to determine the extent to which this chemokine influenced the innate immune response to cancer. To do this, we used the 4T1 murine mammary carcinoma cell line that constitutively expresses CCL2 and generated 4T1 expressing an antisense CCL2 transcript. The antisense-CCL2-expressing 4T1 produced no detectable CCL2. Macrophages from female BALB/c mice were exposed to supernatants from these tumor cells. The results showed that tumor-derived CCL2 was capable of modulating cytokine gene expression but not protein production in resting, activated, and tumor-associated macrophages. In addition, tumor-derived CCL2 did not affect phagocytic activity, nitric oxide production, or cytolytic activity of the macrophages. Overall, these data suggest that tumor-derived CCL2 does not directly influence macrophage-mediated antitumor activity.
\end{abstract}

\section{INTRODUCTION}

CCL2 is produced by many cell types such as T cells, monocytes, and even many tumor cells. It is a potent chemoattractant for monocytes and induces the expression of integrins required for chemotaxis $[1,2]$. Similar to many other members of the CC chemokine family, CCL2 is also a chemoattractant for activated CD4 and $\mathrm{CD}^{+} \mathrm{T}$ cells and natural killer (NK) cells $[3,4]$. Further, it is capable of triggering granule release from NK cells and $\mathrm{CD} 8^{+} \mathrm{T}$ cells and inducing histamine-releasing factor from basophils $[5,6]$. Unfortunately, despite being one of the most frequently investigated chemokines in tumor immunology, the role of tumor-derived CCL2 in tumor immunity remains unclear [7].

Although several studies have reported that introducing the CCL2 gene into tumor cells reduces tumorigenicity and increases monocyte infiltration and cytolysis $[8,9,10,11,12]$, other studies have reported that tumorderived CCL2 correlates with enhanced metastasis, angiogenesis, and tumor progression $[13,14,15]$. The conflicting results indicate a need for further clarification of the inherent paradox that lies within this chemokine's activities. The need to clarify the role of tumor-derived CCL2 in patients with breast cancer is further justified due to the fact that expression may serve as an indicator of early relapse [14].

In an attempt to facilitate the understanding of tumorderived CCL2, we have been using murine mammary carcinoma models. Previously we reported that the murine mammary carcinoma $4 \mathrm{~T} 1$ and spontaneous tumors from rat neu transgenic mice constitutively express CCL2 [16, 17]. Subsequently, we explored the role of tumor-derived CCL2 in the T cell response to the 4T1 mammary carcinoma and found that it was capable of impairing the $\mathrm{T}$ cell response to this tumor [18]. Here, we are interested in determining whether tumor-derived CCL2 influenced macrophage effector function.

For this purpose, we compared the ability of macrophages to respond to $4 \mathrm{~T} 1$ that expressed different levels of CCL2. Thus, resting and activated peritoneal macrophages from $B A L B / c$ mice were exposed to $4 \mathrm{~T} 1$ that expressed normal levels of CCL2 and 4T1 that expressed an antisense CCL2 eukaryotic expression vector which produced no detectable CCL2. The macrophages were then assayed for cytokine gene expression, protein production, as well as phagocytic activity, nitric oxide (NO) production, and cytolytic ability. Collectively, the study revealed that tumor-derived CCL2 could modulate cytokine gene expression but not protein production. Also, tumor-derived CCL2 did not significantly modulate the phagocytic activity, NO production, or the cytolytic activity of the macrophages. Consequently, these data indicate that tumor-derived CCL2 does not directly influence the ability of macrophages to exert antitumor immunity.

\section{MATERIAL AND METHODS}

\section{Mice and tumor cells}

Six to eight week old female BALB/c mice were purchased from the Jackson Laboratory (Bar Harbor, Me) and provided food and water ad libitum. The vector 
transfected control 4T1 tumor cells (A4) and antisense CCL2 transfected 4T1 tumor cells (G7) were created and maintained in complete Roswell Park Memorial Institute (cRPMI) media as previously described [18].

\section{Macrophage isolation and purification}

Activated and resting macrophage populations were used throughout the investigation. Activated macrophages were collected from mice treated with $1 \mathrm{~mL}$ of $3 \%$ thioglycollate (Sigma, St. Louis, Mo) injected into the peritoneal cavity (IP). Four days after injection the mice were sacrificed and $10 \mathrm{~mL}$ of cRPMI were injected into the peritoneal cavity and extracted to collect the peritoneal exudate. Red blood cells were lysed by hypotonic shock. Resting macrophages were collected in a similar manner from untreated mice.

Magnetic cell separation was used to enrich macrophages from the peritoneal exudates. CD11b microbeads (Miltenyi Biotec, Auburn, Calif) were used to do this according to the manufacturer's instructions. The resulting cell population was $92 \%-97 \%$ pure CD $11 b^{+}$macrophages based upon differential cell counts. Differential cell counts were performed by centrifuging $5 \times 10^{4}$ cells onto a slide using a Cytospin (Thermo-Shandon, Pittsburgh, Pa). The cells were stained with the Hema 3 stain kit (Fisher Scientific, Pittsburgh, Pa) and the percent macrophages, neutrophils, and lymphocytes were determined by morphology from three separate fields of view/slide.

\section{Reverse transcriptase-polymerase chain reaction}

To evaluate cytokine gene expression, messenger RNA (mRNA) was isolated using the mRNA isolation kit (Qiagen, Chatsworth, Calif). Complementary DNA (cDNA) was generated using $1 \mu \mathrm{L}$ random hexamer primers (Promega, Madison, Wis), 200 units of M-MLV RT (Promega), and $2 \mathrm{mM} \mathrm{dNTP}$ at $42^{\circ} \mathrm{C}$ for 1 hour. An aliquot $(3 \mu \mathrm{L})$ of the cDNA along with $15 \mu \mathrm{L}$ of primers was then subjected to semiquantitative PCR with taq polymerase $\left(94^{\circ} \mathrm{C}\right.$ for 15 seconds, $59^{\circ} \mathrm{C}$ for 30 seconds, $72{ }^{\circ} \mathrm{C}$ for 45 seconds) for 30 cycles on a thermocycler (MJ Research, Waltham, Mass). The cytokine-specific primers were synthesized by Integrated DNA Technologies (Coralville, Iowa):

(i) GAPDH (212 bp product size) forward 5' -CAGGTTGTCTCCTGCGACTT- $3^{\prime}$ reverse $5^{\prime}$-CTTGCTCAGTGTCCTTGCTG-3',

(ii) IL-12 p40 subunit (482 bp product size) forward 5'-GGAAGCACGGCAGCAGAATAA-3' reverse $5^{\prime}$-AGCCAACCAAGCAGAAGACAG-3',

(iii) $\mathrm{TNF}-\alpha$ (283 bp product size) forward 5' -CCAGGAGGGAGAACAGAAACT-3' reverse 5' -CAGTAGACAGAAGAGCGTGGTG-3',

(iv) IL-18 (219 bp product size) forward $5^{\prime}$-CTGGCTGTGACCCTCTCTGT-3' reverse 5'-AGCATCATCTTCCTTTTGGC-3'.

RT-PCR products were analyzed on a $2 \%$ agarose gel with ethidium bromide (Sigma), and analyzed using a gel documentation system (Alpha Innotech Corp, San Leandro, Calif).

\section{Cytokine and NO production}

To evaluate cytokine and NO production, macrophages were exposed to A4 (CCL2+) and G7 (CCL2-) tumor supernatants. The A4 and G7 supernatants were obtained by plating $1 \times 10^{6}$ cells/well in a 24 -well flat-bottom cell culture plate (BD Falcon, Franklin Lakes, NJ) in $1 \mathrm{~mL}$ of cRPMI. The supernatants were collected after a 24hour incubation at $37^{\circ} \mathrm{C}$ and $5.0 \% \mathrm{CO}_{2}$, centrifuged for 5 minutes at $350 \mathrm{xg}$, transferred to sterile microfuge tubes, and stored at $-20{ }^{\circ} \mathrm{C}$. Macrophages were then plated at $1 \times 10^{6}$ cells/well in $1 \mathrm{~mL}$ of either the A4 or G7 tumor supernatant and incubated at $37^{\circ} \mathrm{C}$ for 24 hours. Following the incubation, supernatants were harvested and assayed for cytokines by specific ELISA or NO content according to manufacturer's instructions (R\&D Systems, Minneapolis, Minn). Messenger RNA was isolated from the same macrophages and used to examine cytokine gene expression.

For analysis of tumor-associated macrophages (TAM), four-week subcutaneous A4 and G7 tumors were harvested and digested in a collagenase cocktail ( $1 \mathrm{mg} / \mathrm{mL}$ collagenase type IV, $20 \mu \mathrm{g} / \mathrm{mL}$ DNase, $10 \mathrm{U} / \mathrm{mL}$ hyaluronidase). Macrophages were enriched from the tumor digest as described above for the peritoneal macrophages and cultured at $1 \times 10^{6}$ cells/well. Twentyfour-hour supernatants from the TAM were assayed for cytokine production by ELISA.

\section{Phagocytic activity}

To examine phagocytic activity, E coli BioParticles (Molecular Probes Inc, Eugene, Ore) were reconstituted in $2 \mathrm{mM}$ sodium azide and phosphate-buffered saline (PBS) at $20 \mathrm{mg} / \mathrm{mL}$. E coli and opsonizing reagent (Molecular Probes) were added in equal volumes to a microfuge tube, vortexed, and incubated at $37^{\circ} \mathrm{C}$ for 1 hour to allow adequate conjugation of antibodies to $E$ coli. After being washed with PBS, the opsonized $E$ coli were counted and resuspended in PBS at $1 \times 10^{8}$ cells $/ \mathrm{mL}$. Next, activated macrophages cultured in $1 \mathrm{~mL}$ of A4 or G7 supernatants for 24 hours were added onto $12 \mathrm{~mm}$ round poly-L-lysine coated coverslips (Becton Dickinson, Bedford, Mass). Labeled BioParticles, $1 \times 10^{6} \mathrm{E}$ coli, were added directly to the wells at a $10: 1$ ratio (E coli : macrophage). Following a 30-minute incubation at $37^{\circ} \mathrm{C}$, nonphagocytosed $E$ coli were washed away with PBS and the coverslips were prepared for viewing by confocal microscopy using slowfade antifade reagent (Molecular Probes, Eugene, Ore). Differential phagocytic activity was measured from three separate fields of view/slide.

\section{Cytolytic activity}

In order to measure the cytolytic ability of activated macrophages exposed to tumor-derived CCL2, macrophages were resuspended in $1 \mathrm{~mL}$ of either $\mathrm{A} 4$ or 


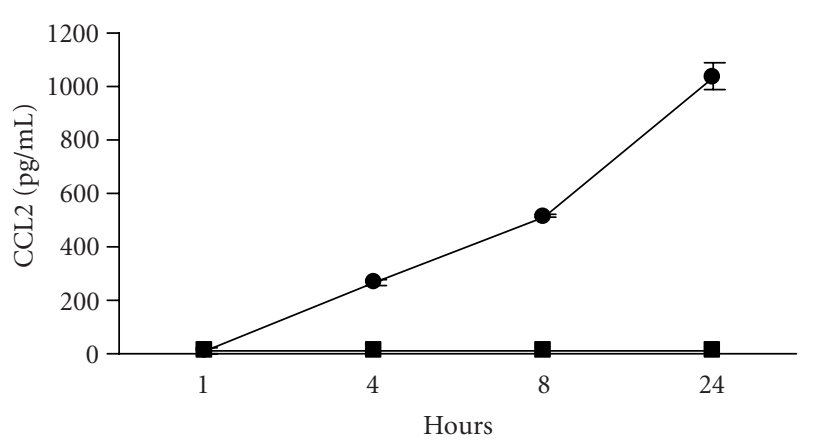

$\underset{-\mathrm{G} \text { G7 }}{\longrightarrow}$

FIgURE 1. Tumor-derived CCL2 production. Supernatants were taken from A4 and G7 tumor cells at different time points and evaluated for CCL2 production by ELISA. The data are representative of three separate experiments with standard deviation shown.

G7 supernatants at $1 \times 10^{5}$ cells $/ \mathrm{mL}$. These cell suspensions were placed in separate wells, in a 24 -well culture plate, with $1 \times 10^{3} 4 \mathrm{~T} 1$ tumor cells. Following a 72-hour incubation at $37^{\circ} \mathrm{C}, 5 \% \mathrm{CO}_{2}$, the tumor cells were removed by trypsinization. The macrophages which are resistant to trypsin remained in the wells. The surviving tumor cells were quantified using trypan blue exclusion, and data were reported as percent killing compared to control wells without macrophages.

\section{Statistical analysis}

All data are presented as a mean plus or minus the standard deviation of the mean. Statistical comparisons were made using a paired Student's $t$ test with a one-tailed distribution.

\section{RESULTS}

\section{Macrophages and tumor cells}

For this study, activated and resting macrophages were collected from mice and exposed to supernatants from $4 \mathrm{~T} 1$ that produced normal levels of CCL2 (A4) or 4T1 that lacked CCL2 expression (G7). CCL2 expression by these cells is shown in Figure 1. The A4 cells produced $1000 \mathrm{pg} / \mathrm{mL}$ of the chemokine over a 24-hour period, while CCL2 levels were below detection $(<15 \mathrm{pg} / \mathrm{mL})$ for G7 cells. Previously we reported that $4 \mathrm{~T} 1$ produced approximately $850 \mathrm{pg} / \mathrm{mL}$ of CCL2 [16]. Macrophages collected naïve mice (resting macrophages) and mice that received thioglycollate (activated macrophages) were easily distinguishable based upon cell size. Although the tumorderived CCL2 had no distinguishable effect on morphology, the activated macrophages were consistently larger than the resting macrophages (data not shown).

\section{CCL2 associated alterations in cytokine expression}

To evaluate whether tumor-derived CCL2 influenced cytokine gene expression in macrophages, semiquantita- tive RT-PCR was used. For these experiments, mRNA was isolated from macrophages exposed to supernatants from A4 and G7 tumor cells to analyze IL-12, IL-18, and TNF- $\alpha$ gene expression (Figure 2). Densitometric analysis revealed an increase in IL-18, and TNF- $\alpha$ expression from both activated and resting macrophages exposed to A4 supernatants compared to those exposed to G7 supernatants, while IL-12 expression was not detected (Figure 2).

In order to determine whether the altered gene expression correlated with protein production, supernatants from macrophages exposed to A4 and G7 supernatants were harvested and assayed for cytokine levels by ELISA. The data revealed no difference in TNF- $\alpha$ levels while IL-18 and IL-12 were both below detection levels of the ELISA, $25 \mathrm{pg} / \mathrm{mL}$ for IL- 18 and $4 \mathrm{pg} / \mathrm{mL}$ for IL-12 (Figure 3a). Accordingly, gene expression did not correlate with protein expression for TNF- $\alpha$ and IL-18.

To determine whether higher concentrations of CCL2 could influence cytokine production from the macrophages, recombinant CCL2 (rCCL2) was used. Surprisingly, IL-12 production showed a dose-dependent relationship to rCCL2, whereas TNF- $\alpha$ did not (Figure 3b). IL-18 levels were still below detection. For both TNF$\alpha$ and IL-12, the resting and activated macrophages responded similarly to rCCL2. Consequently, tumorderived CCL 2 could enhance TNF- $\alpha$ production, but IL12 was only induced with rCCL2.

In order to address whether a longer exposure to tumor-derived CCL2 could influence cytokine expression, we assayed cytokine production from TAM. For this purpose, TAM from four-week A4 and G7 tumors growing in mice were evaluated for IL-12, IL-18, and TNF- $\alpha$ production. After isolation and enrichment (70\%-75\% pure $\mathrm{CD}_{11} \mathrm{~b}^{+}$macrophages), all three cytokines (IL-12 20 pg/mL, IL-18 40 pg/mL, TNF- $\alpha 800$ pg/mL) were detected from the TAM. However, similar levels were found in the A4 and G7 tumors indicating that tumor-derived CCL2 did not influence their expression (Figure 3c).

\section{CCL2 associated alterations in effector function}

$\mathrm{NO}$ is a major secretory product of mammalian cells that initiates host defense [19]. The amount of NO secreted by macrophages is thus a reliable measure of macrophage effector function. Supernatants from macrophages incubated with A4 and G7 typically contained $270 \mu \mathrm{M}$ of $\mathrm{NO}$ (Figure $4 \mathrm{a}$ ). The amount of NO produced did not change significantly whether the macrophages were activated or naïve, nor whether they were exposed to A4 or G7 tumor supernatants (Figure 4a). As a result, tumor-derived CCL2 did not modulate NO production by the macrophages.

To determine whether tumor-derived CCL2 influenced macrophage cytolytic activity, a cytotoxicity assay was used. To measure the killing activity of 4T1 carcinoma cells, activated macrophages were incubated with the tumor cells and either A4 or G7 supernatants for 72 


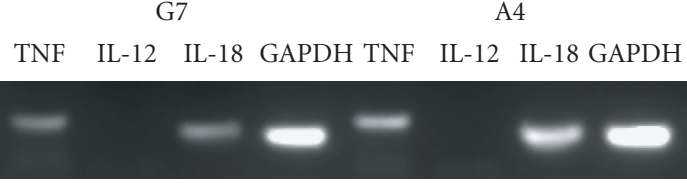

(a)

G7

A4

TNF IL-12 IL-18 GAPDH TNF IL-12 IL-18 GAPDH

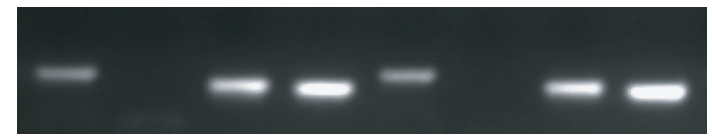

(b)

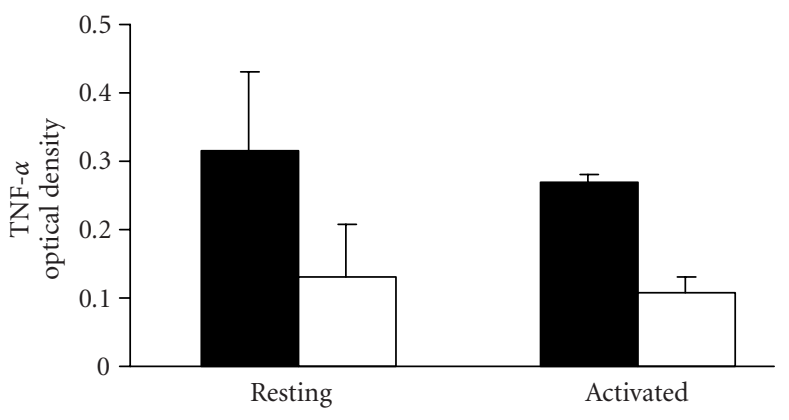

A4

$\square$ G7

(c)

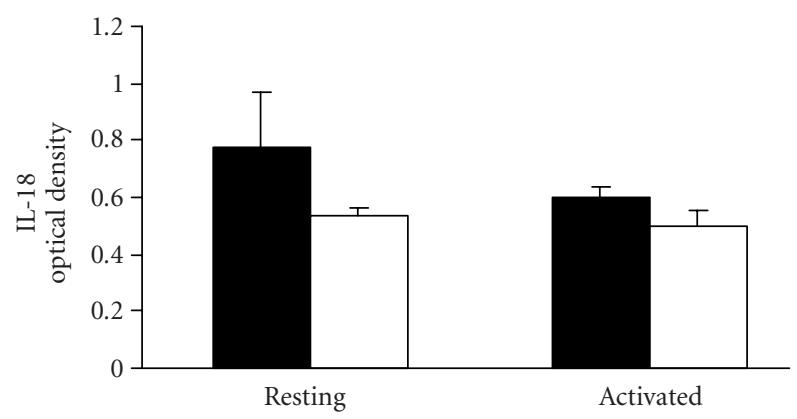

a4 4

$\square$ G7

(d)

Figure 2. Cytokine analysis by RT-PCR. Resting (a) and activated (b) macrophages were examined for IL-12, IL-18, and TNF- $\alpha$ expression. GAPDH was used as a positive control. The data represent one of three separate experiments. For the densitometric analysis, the optical densities were calculated by comparison to the positive control (GAPDH).

hours. There was no significant difference in tumor cytolysis by the macrophages (Figure 4b). Percent killing activity of $4 \mathrm{~T} 1$ tumor cells in the presence or absence of

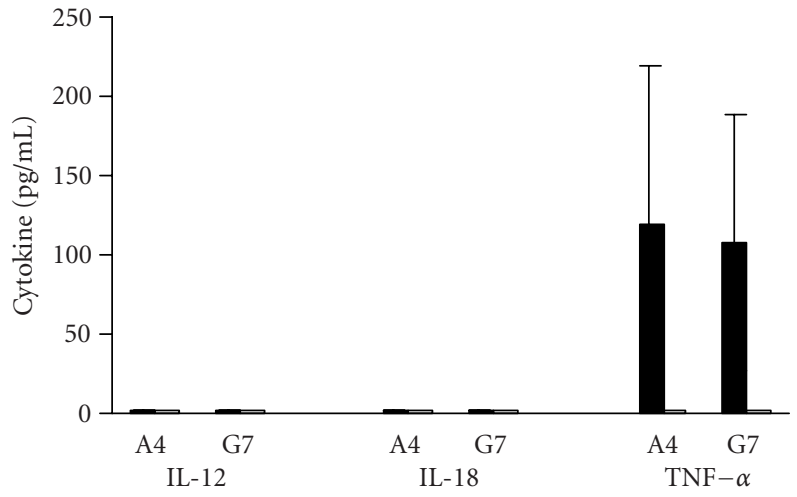

Resting

Activated

(a)

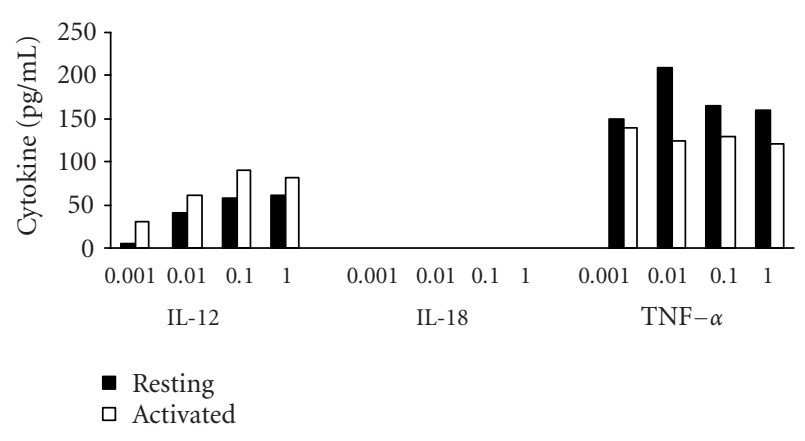

(b)

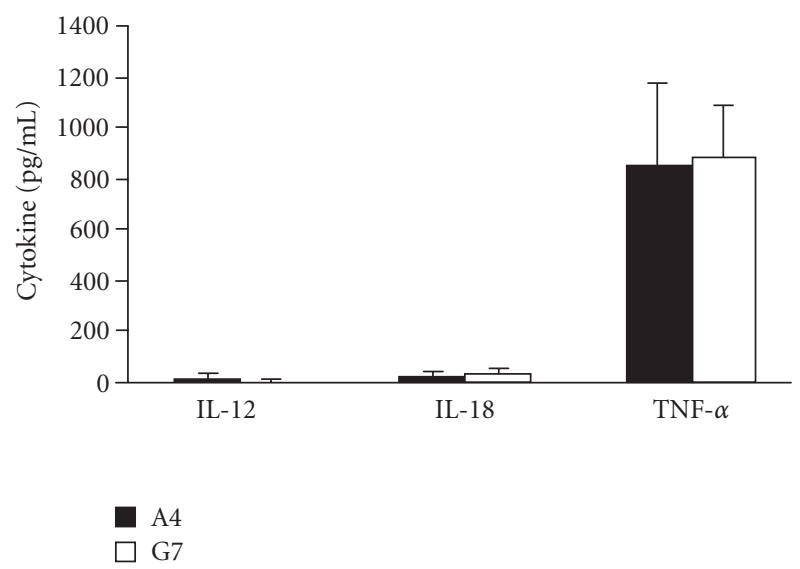

(c)

FIGURE 3. Cytokine production by ELISA. (a) Resting and activated macrophages were examined for cytokine production after exposure to A4 and G7 supernatants. The data are representative of three separate experiments. The error bars represent the standard deviation of the mean of duplicate wells analyzed by ELISA. (b) Following exposure of macrophages to rCCL2 ( $\mu \mathrm{g} / \mathrm{mL})$, supernatants were assayed for the same cytokines. (c) TAM harvested from four-week A4 and G7 tumors were analyzed for cytokine production. The data shown are representative of three separate experiments, with error bars denoting the standard deviation from the mean. 


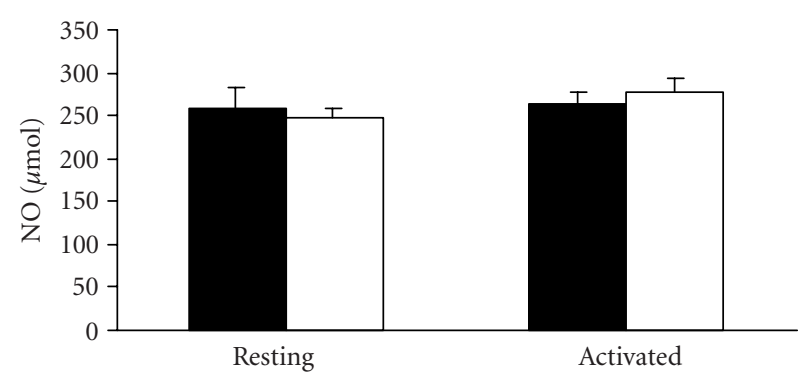

(a)

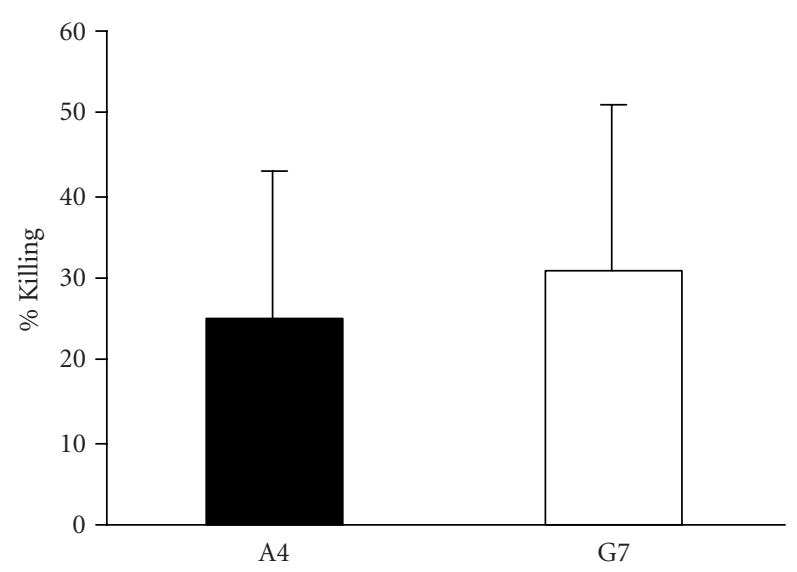

(b)

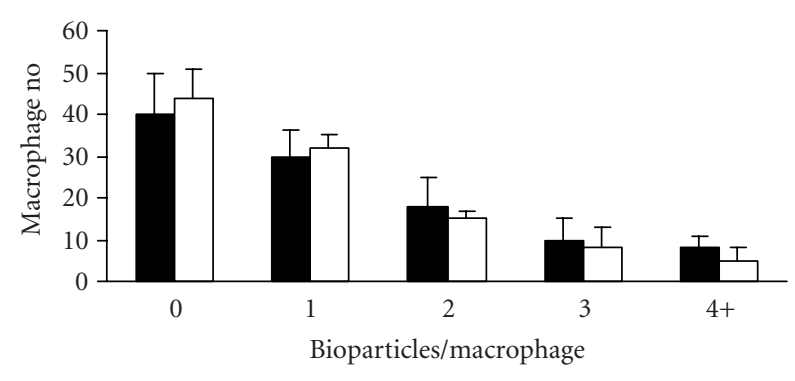

(c)

Figure 4. Macrophage effector function. (a) Macrophages exposed to A4 ( $\square)$ and G7 ( $\square$ ) supernatants were evaluated for total NO production by measuring the concentration of nitrite. The amount of nitrite was measured in three separate experiments from duplicate wells by ELISA. Error bars represent the standard deviation of the mean. (b) Macrophages were exposed to A4 and G7 supernatants for 72 hours to test their ability to modulate cytolytic activity. The figure represents macrophage-mediated cytolysis of the tumor cells. The data are representative of three separate cell counts/experiment, with error bars showing standard deviation from the mean. (c) The ability of macrophages exposed to A4 $(\square)$ and G7 ( $\square$ ) supernatants to phagocytose $E$ coli was determined through confocal microscopy. Using both light and florescence settings simultaneously, it was possible to distinguish phagocytosed bacteria. The data are representative of two separate experiments where cell counts were determined from three separate fields of view/slide. Error bars represent standard deviation from the mean. tumor-derived CCL2 was 25\%-30\%. These data indicated that tumor-derived CCL2 did not modulate the cytolytic ability of the macrophages.

Finally, we investigated whether tumor-derived CCL2 could influence the phagocytic activity of macrophages. For this purpose, we assayed the ability of activated macrophages to phagocytose fluorescently labeled E coli BioParticles (Figure 4c). Differential phagocytosis was measured by counting individual bacteria phagocytosed in three separate fields of view/slide. The data indicated no difference in the phagocytic ability of macrophages exposed to supernatants from A4 compared to G7 tumors. The number of $E$ coli engulfed by the macrophages after exposure to A4 and G7 supernatants is shown in Figure 4c. A comparison of phagocytosis based upon whether the macrophages engulfed any E coli, regardless of number, also showed no difference (data not shown). Therefore, tumor-derived CCL2 did not influence macrophage phagocytic activity.

\section{DISCUSSION}

As one of the first chemokines used to genetically modify tumor cells, CCL2 has been investigated in a number of different models. For instance, it was found that chinese hamster ovary $(\mathrm{CHO})$ cells transfected with either human or murine CCL2 gene lose their ability to form tumors in nude mice [20]. Similar results were obtained when CCL2 expressing cells were injected with nonexpressing $\mathrm{CHO}$ or HeLa cells [20]. When the B78/HI melanoma cell line was transfected with the CCL2 gene, a significant delay in tumor growth was observed in syngeneic and nude mice [21].

Whether CCL2 expression could affect metastatic potential and macrophage susceptibility has also been investigated. Huang et al [9] reported that CCL2 decreased the tumorigenicity and metastatic potential of the CT26 cell line. The CCL2 expressing tumors were also highly susceptible to lysis by lipopolysaccharide (LPS) stimulated macrophages [9]. The same group showed similar findings using the murine renal adenocarcinoma cell line RENCA [10]. Another group used the C20 colon carcinoma line and reported that the combination of CCL2 gene transfection and LPS delivery enhanced antitumor immunity [11]. However, since CCL2 expression has been correlated with progression in patients with breast cancer, a role for CCL2 in increasing tumorigenicity has also been hypothesized [14]. One of the proposed mechanisms by which CCL2 could enhance tumorigenesis is by enhancing the synthesis of macrophage-derived TNF$\alpha$ since TNF- $\alpha$ has been shown to stimulate angiogenesis $[22,23]$. In fact, there is evidence for such an association in a murine model. A comparison of two related murine mammary adenocarcinoma cell lines revealed a direct correlation between tumorigenicity and CCL2 expression [15]. The Ly-6hi DA3 cell line expressed high 
levels of CCL2 and was more malignant than the Ly-6lo DA3 cell line that expressed lower levels of CCL2.

Here we investigated whether tumor-derived CCL2 could directly influence macrophage effector function. We report that tumor-derived CCL2 is capable of modulating cytokine gene expression, but not cytokine production in murine peritoneal macrophages obtained from BALB/c mice. The fact that cytokine production was not modulated by tumor-derived CCL2 was surprising and contradictory to several other reports. Our data may differ from other reports due to the fact that we did not use LPS to stimulate the macrophages. For instance, Seki et al [24] reported $600 \mathrm{pg} / \mathrm{mL}$ of IL-18 produced by Kupffer cells stimulated with LPS, and TNF- $\alpha$ levels of approximately $3000 \mathrm{pg} / \mathrm{mL}$. The IL-12 results were also interesting because $1000 \mu \mathrm{g} / \mathrm{mL}$ of rCCL2 induced IL-12 expression, whereas tumor-derived CCL2 (also at $1000 \mu \mathrm{g} / \mathrm{mL}$ ) did not. It is interesting to speculate that there is another factor produced by these tumors that suppresses the IL-12 production and thus counters the effect of tumor-derived CCL2.

The cytotoxicity data also contrasted with previous reports that CCL2 modulates macrophage mediated cytotoxicity [9]. Again, our results may differ from others because we did not use LPS to activate the macrophages. Also, another study reported that modulation of tumorderived CCL2 did not influence monocyte mediated cytotoxicity in a significant manner. Asano et al [25] inserted the CCL2 gene or antisense transcript into two human brain tumor cell lines; HBT28, which constitutively expressed high levels of CCL2, and HBT20, which expressed lower levels of CCL2. Decreasing and increasing CCL2 expression in the tumor cells had a similar effect on monocyte mediated cytotoxicity.

Ultimately, this investigation has shown that tumorderived CCL2 may induce cytokine expression at the mRNA level, but does not affect protein production. Moreover, tumor-derived CCL2 did not directly influence the effector function of the macrophages. The chemokine was not able to modulate NO production, phagocytosis, or tumor cytolysis. Consequently, the exclusive function of tumor-derived CCL2 may be in aiding angiogenesis as others have suggested $[13,22]$.

\section{ACKNOWLEDGMENTS}

This study was funded in part by a grant from the Department of Defense Breast Cancer Research Program DAMD17-01-1-0288. We would also like to thank the Department of Biology and Lafayette College for supporting this work.

\section{REFERENCES}

[1] Martinet N, Beck G, Bernard V, et al. Mechanism for the recruitment of macrophages to cancer site. In vivo concentration gradient of monocyte chemotactic activity. Cancer. 1992;70(4):854-860.
[2] Vaddi K, Newton RC. Regulation of monocyte integrin expression by beta-family chemokines. J Immunol. 1994;153(10):4721-4732.

[3] Carr MW, Roth SJ, Luther E, Rose SS, Springer TA. Monocyte chemoattractant protein 1 acts as a T-lymphocyte chemoattractant. Proc Natl Acad Sci USA. 1994;91(9):3652-3656.

[4] Allavena P, Bianchi G, Zhou D, et al. Induction of natural killer cell migration by monocyte chemotactic protein-1, -2 and -3. Eur J Immunol. 1994;24(12):3233-3236.

[5] Loetscher P, Seitz M, Clark-Lewis I, Baggiolini M, Moser B. Activation of NK cells by CC chemokines. Chemotaxis, $\mathrm{Ca} 2+$ mobilization, and enzyme release. J Immunol. 1996;156(1):322-327.

[6] Kuna P, Reddigari SR, Rucinski D, Oppenheim JJ, Kaplan AP. Monocyte chemotactic and activating factor is a potent histamine-releasing factor for human basophils. J Exp Med. 1992;175(2):489-493.

[7] Brault MS, Kurt RA. Chemokines and antitumor immunity: walking the tightrope. Int Rev Immunol. 2003;22(3-4):199-228.

[8] Rollins BJ, Sunday ME. Suppression of tumor formation in vivo by expression of the JE gene in malignant cells. Mol Cell Biol. 1991;11(6):3125-3131.

[9] Huang S, Singh RK, Xie K, et al. Expression of the $J E / M C P-1$ gene suppresses metastatic potential in murine colon carcinoma cells. Cancer Immunol Immunother. 1994;39(4):231-238.

[10] Huang S, Xie K, Singh RK, Gutman M, Bar-Eli M. Suppression of tumor growth and metastasis of murine renal adenocarcinoma by syngeneic fibroblasts genetically engineered to secrete the JE/MCP-1 cytokine. J Interferon Cytokine Res. 1995;15(7):655665.

[11] Nakashima E, Kubota Y, Matsushita R, et al. Synergistic antitumor interaction of human monocyte chemotactant protein-1 gene transfer and modulator for tumor-infiltrating macrophages. Pharm Res. 1998;15(5):685-689.

[12] Nokihara H, Nishioka Y, Yano S, et al. Monocyte chemoattractant protein-1 gene modification of multidrug-resistant human lung cancer enhances antimetastatic effect of therapy with anti-Pglycoprotein antibody in SCID mice. Int J Cancer. 1999;80(5):773-780.

[13] Opdenakker G, Van Damme J. Cytokines and proteases in invasive processes: molecular similarities between inflammation and cancer. Cytokine. 1992;4(4):251-258.

[14] Ueno T, Toi M, Saji H, et al. Significance of macrophage chemoattractant protein-1 in macrophage recruitment, angiogenesis, and survival in human breast cancer. Clin Cancer Res. 2000;6(8): 32823289.

[15] Neumark E, Anavi R, Witz IP, Ben-Baruch A. MCP-1 expression as a potential contributor to the high malignancy phenotype of murine 
mammary adenocarcinoma cells. Immunol Lett. 1999;68(1):141-146.

[16] Kurt RA, Baher A, Wisner KP, Tackitt S, Urba WJ. Chemokine receptor desensitization in tumorbearing mice. Cell Immunol. 2001;207(2):81-88.

[17] Kurt RA, Bauck M, Harma S, et al. Altered chemokine receptor sensitivity in FVBN202 rat neu transgenic mice. Breast Cancer Res Treat. 2003;77(3):225232.

[18] Vitiello PF, Shainheit MG, Allison EM, Adler EP, Kurt RA. Impact of tumor-derived CCL2 on T cell eEffector function. Immunol. Lett. 2004;91:(23):239-245.

[19] Stuehr DJ, Marletta MA. Mammalian nitrate biosynthesis: mouse macrophages produce nitrite and nitrate in response to Escherichia coli lipopolysaccharide. Proc Natl Acad Sci USA. 1985;82:7738-7742.

[20] Rollins BJ, Sunday ME. Suppression of tumor formation in vivo by expression of the JE gene in malignant cells. Mol Cell Biol. 1991;11(6):3125-3131.

[21] Bottazzi B, Walter S, Govoni D, Colotta F, Mantovani A. Monocyte chemotactic cytokine gene transfer modulates macrophage infiltration, growth, and susceptibility to IL-2 therapy of a murine melanoma. J Immunol. 1992;148(4):1280-1285.

[22] Mantovani A. Tumor-associated macrophages in neoplastic progression: a paradigm for the in vivo function of chemokines. Lab Invest. 1994;71(1):516.

[23] Leibovich SJ, Polverini PJ, Shepard HM, Wiseman DM, Shively V, Nuseir N. Macrophage-induced angiogenesis is mediated by tumour necrosis factoralpha. Nature. 1987;329(6140):630-632.

[24] Seki E, Tsutsui H, Nakano H, et al. Lipopolysaccharide-induced IL-18 secretion from murine Kupffer cells independently of myeloid differentiation factor 88 that is critically involved in induction of production of IL-12 and IL-1beta. J Immunol. 2001;166(4):2651-2657.

[25] Asano T, An T, Jia SF, Kleinerman ES. Altered monocyte chemotactic and activating factor gene expression in human glioblastoma cell lines increased their susceptibility to cytotoxicity. J Leukoc Biol. 1996;59(6):916-924.

* Corresponding author.

E-mail: kurtr@lafayette.edu

Fax: +1 610330 5705; Tel: +1 6103305681 

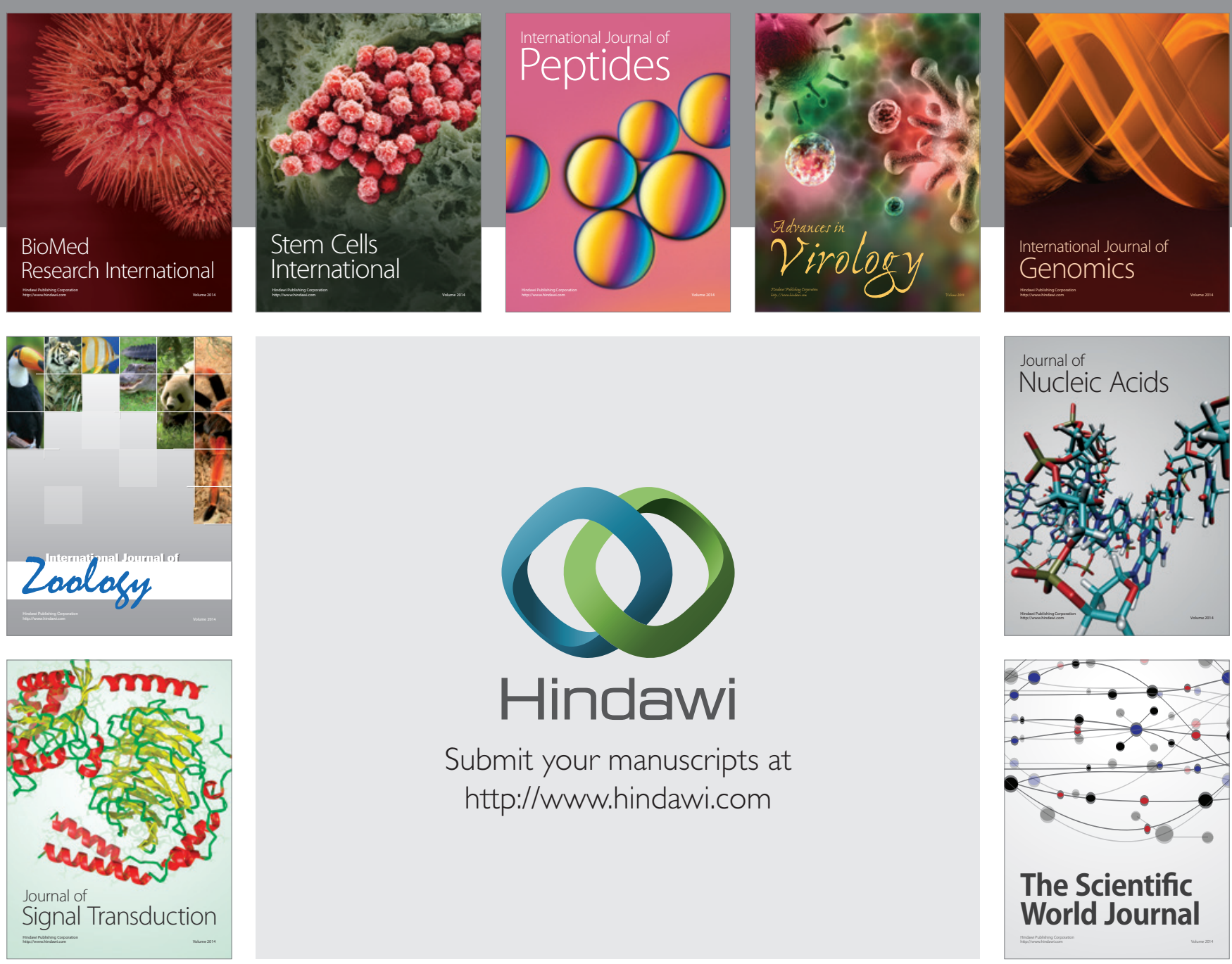

Submit your manuscripts at

http://www.hindawi.com
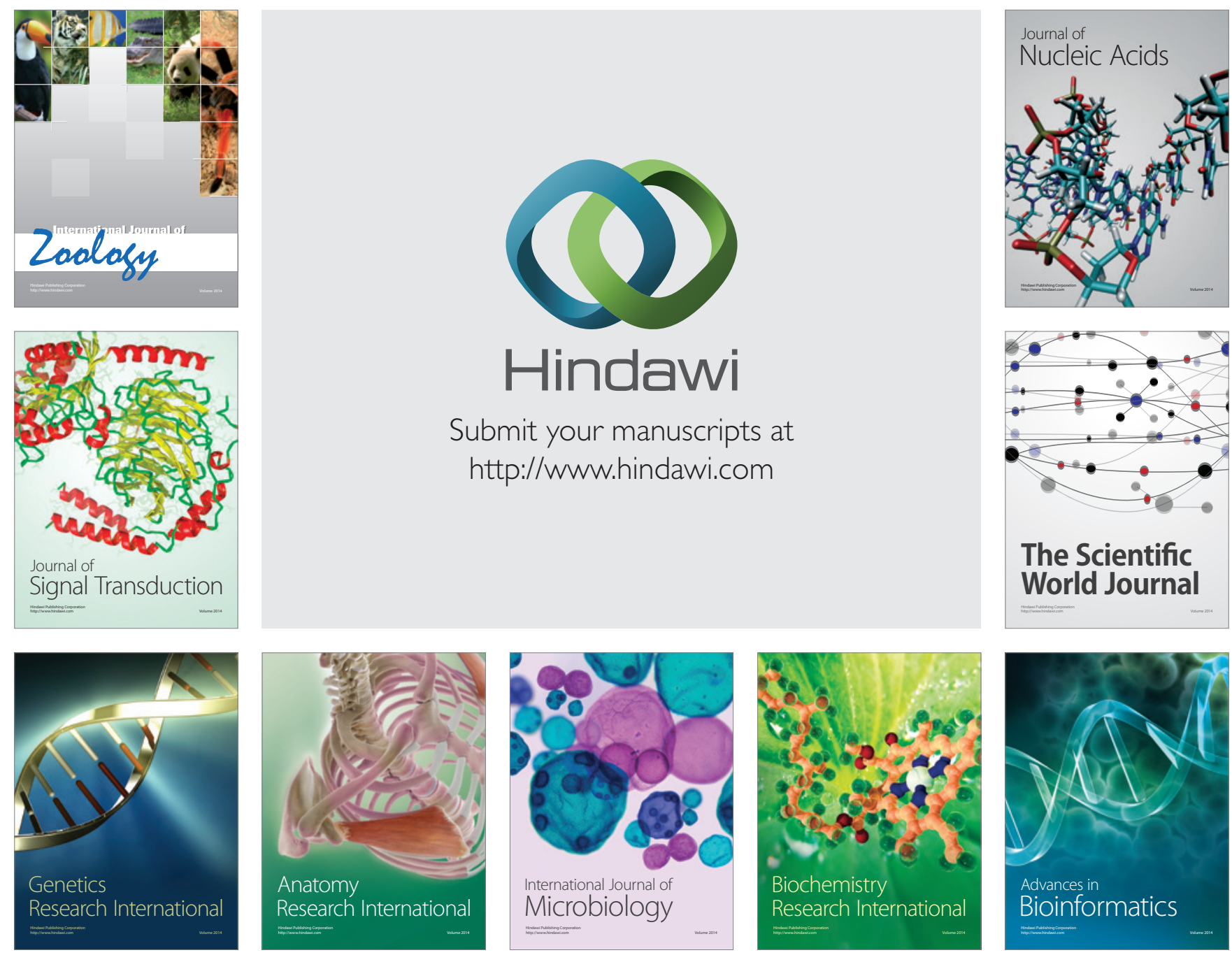

The Scientific World Journal
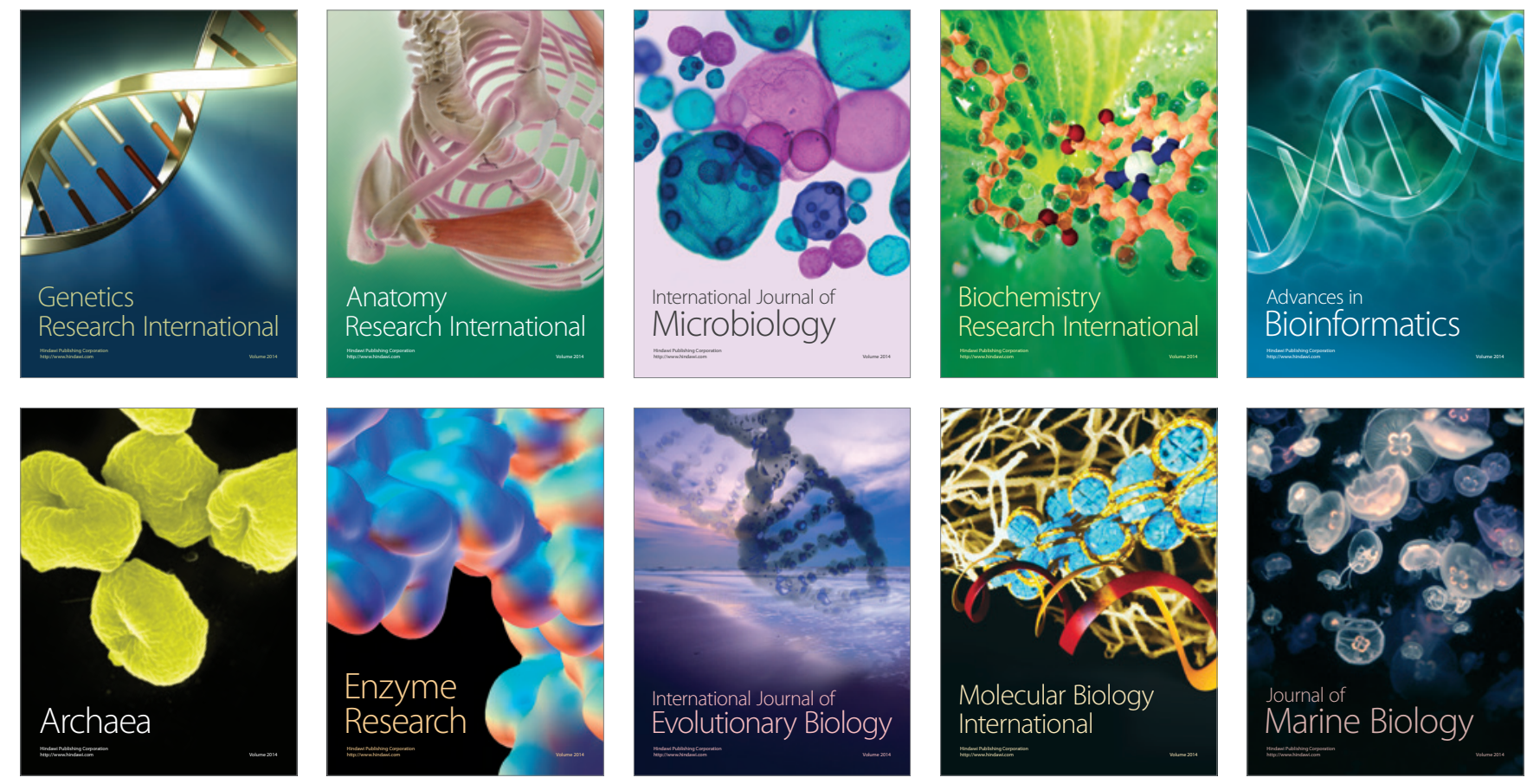